\title{
Characterization of a thiamin diphosphate-dependent phenylpyruvate decarboxylase from Saccharomyces cerevisiae
}

Malea M. Kneen ${ }^{1}$, Razvan Stan ${ }^{1}$, Alejandra Yep ${ }^{2}$, Ryan P. Tyler ${ }^{2}$, Choedchai Saehuan $^{2, *}$ and Michael J. McLeish ${ }^{1}$

1 Department of Chemistry and Chemical Biology, Indiana University-Purdue University Indianapolis, IN, USA

2 Department of Medicinal Chemistry, University of Michigan, Ann Arbor, MI, USA

\section{Keywords}

amino acid catabolism; Ehrlich pathway: homology model; mutagenesis; TPP

\section{Correspondence}

M. J. McLeish, Department of Chemistry and Chemical Biology, Indiana UniversityPurdue University Indianapolis, 402 North Blackford Street, Indianapolis, IN 46202, USA

Fax: +1 3172744701

Tel: +13172746889

E-mail: mcleish@iupui.edu

\section{*Present address}

Department of Medical Technology, Faculty of Allied Health Sciences, Naresuan University, Phitsanulok Thailand

(Received 23 December 2010, revised 7 March 2011, accepted 21 March 2011)

doi:10.1111/j.1742-4658.2011.08103.x
The product of the ARO10 gene from Saccharomyces cerevisiae was initially identified as a thiamine diphosphate-dependent phenylpyruvate decarboxylase with a broad substrate specificity. It was suggested that the enzyme could be responsible for the catabolism of aromatic and branchedchain amino acids, as well as methionine. In the present study, we report the overexpression of the ARO10 gene product in Escherichia coli and the first detailed in vitro characterization of this enzyme. The enzyme is shown to be an efficient aromatic 2-keto acid decarboxylase, consistent with it playing a major in vivo role in phenylalanine, tryptophan and possibly also tyrosine catabolism. However, its substrate spectrum suggests that it is unlikely to play any significant role in the catabolism of the branched-chain amino acids or of methionine. A homology model was used to identify residues likely to be involved in substrate specificity. Site-directed mutagenesis on those residues confirmed previous studies indicating that mutation of single residues is unlikely to produce the immediate conversion of an aromatic into an aliphatic 2-keto acid decarboxylase. In addition, the enzyme was compared with the phenylpyruvate decarboxylase from Azospirillum brasilense and the indolepyruvate decarboxylase from Enterobacter cloacae. We show that the properties of the two phenylpyruvate decarboxylases are similar in some respects yet quite different in others, and that the properties of both are distinct from those of the indolepyruvate decarboxylase. Finally, we demonstrate that it is unlikely that replacement of a glutamic acid by leucine leads to discrimination between phenylpyruvate and indolepyruvate, although, in this case, it did lead to unexpected allosteric activation.

\section{Introduction}

The Ehrlich pathway, which permits the use of leucine, isoleucine, valine, methionine, tyrosine, tryptophan or phenylalanine as a sole nitrogen source, leads to the formation of the fusel alcohols and acids (Fig. 1) [1]. Indeed, in Saccharomyces cerevisiae, the Ehrlich pathway is the only route for phenylalanine and

\footnotetext{
Abbreviations

BFDC, benzoylformate decarboxylase; IPDC, indole-3-pyruvate decarboxylase; IPyA, indole-3-pyruvic acid; KdcA, keto acid decarboxylase; PDB, Protein Data Bank; PDC, pyruvate decarboxylase; PPA, phenylpyruvic acid; PPDC, phenylpyruvate decarboxylase; ThDP, thiamin diphosphate.
} 


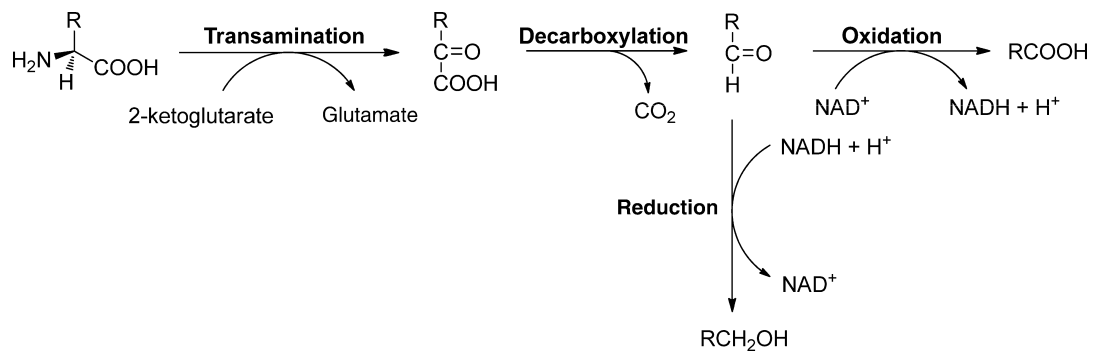

Fig. 1. The Ehrlich pathway: catabolism of amino acids to produce alcohols or carboxylic acids.

tryptophan catabolism [2]. The amino acids are initially transaminated to 2-keto acids and, in the subsequent irreversible step, the 2-keto acids are decarboxylated by any of several thiamin diphosphate (ThDP)-dependent decarboxylases. Depending on the redox state of the cell [3], the resulting aldehydes are then converted by a suite of alcohol and aldehyde dehydrogenases to fusel alcohols or acids, respectively [4]. The fusel products are finally excreted into the surrounding medium. In yeast-fermented foods and beverages, these products are important contributors to flavors, both desirable and undesirable [1]. Recently, interest in this pathway has extended to a potential role in the production of biofuels. Branched-chain alcohols have significant advantages, such as higher energy density and lower hygroscopicity, over ethanol [5]. Consequently, there is considerable attention being paid to those enzymes that are involved in the production of branched-chain 2-keto acids [5,6].

S. cerevisiae expresses several ThDP-dependent decarboxylases that permit the assimilation of the aromatic and branched-chain amino acids [7-9]. The three pyruvate decarboxylase (PDC) isoforms, PDC1 [10], PDC5 [11] and PDC6 [12], are the best-studied of these enzymes. The presence of PDC-independent decarboxylase activity in $S$. cerevisiae was suggested by the ability of PDC-deletion strains to metabolize both branched-chain and aromatic amino acids [8]. This ability was traced to the $A R O 10$ gene, also known as YDR380w, which was induced by tryptophan through the same mechanism as ARO9, the gene encoding aromatic aminotransferase II [13]. The latter enzyme catalyzes the first step in aromatic amino acid catabolism and is induced by aromatic amino acids via the transcription factor Aro80 [13]. Subsequently, ARO10 was found to be inducible by growth on phenylalanine as a sole nitrogen source, and it was shown that the ARO10 gene product, ScPPDC, was a phenylpyruvate decarboxylase with a broad substrate specificity [4]. In addition to being upregulated by methionine and leucine [4], transcriptome analyses of $S$. cerevisiae cells grown on various nitrogen sources showed that isoleucine and threonine also trigger transcription of Aro80 target genes [14]. Therefore, potentially, ARO10 and its associated genes may be responsible for the catabolism of aromatic and branched-chain amino acids, as well as methionine. However, ScPPDC is essentially unable to utilize pyruvate [4].

As part of an ongoing project in our laboratory, we are interested in determining the rules that govern substrate specificity in ThDP-dependent decarboxylases. In the first instance, we focused on converting benzoylformate decarboxylase (BFDC) into a PDC, which involved shifting the enzyme's preference from binding a phenyl group to binding a methyl group $[15,16]$. Although the results obtained from those studies have been promising, with a 11000 -fold improvement in pyruvate utilization by BFDC [16], the experimental design has been complicated by differences in the position of the catalytic residues in the two decarboxylases [17]. ScPPDC is more similar to S. cerevisiae PDC1 (32\% identity, $45 \%$ similarity) than is BFDC $(18 \%$ identity, $33 \%$ similarity), and it was considered that ScPPDC may prove to be a more tractable subject for conversion to a PDC. ScPPDC may also be considered an attractive target for manipulation of the production of fusel alcohols for the food, cosmetics and biofuel industries [1], as well as being useful for stereospecific carboligation reactions [18].

In the present study, we report the overexpression of the ARO10 gene product in Escherichia coli and the first detailed in vitro characterization of this enzyme (UniProt ID: Q06408). The initial steps towards understanding the factors influencing decarboxylation of shorter chain-length substrates are also reported. Finally, we test the proposal [19] that a single residue may be used to differentiate between the phenylpyruvate decarboxylases and the indolepyruvate decarboxylases (IPDCs).

\section{Results and Discussion}

The ARO10 gene product has been identified as a phenylpyruvate decarboxylase with a broad spectrum of activity $[3,4]$. PPDCs have been reported in a range of bacterial species, including Achromobacter eurydice 
[20], Acinetobacter calcoaceticus [21], Pseudomonas putida [22], Nocardia sp. 239 [23] and Thauera aromatica $[23,24]$, although none of these enzymes has been characterized in detail. PPDC activity has also been observed in crude extracts of Aspergillus niger [25] and BLAST searches [26] indicate that PPDCs are likely to exist in other fungal species. Similar to the latter enzymes, at approximately $72 \mathrm{kDa}$, the $S c P P D C$ is predicted to be almost $20 \%$ larger than most ThDPdependent decarboxylases. Nonetheless, the signature features of a ThDP-dependent enzyme are maintained.

In the present study, the gene encoding $S c P P D C$ was amplified from $S$. cerevisiae genomic DNA and visualized as an approximately $1.9 \mathrm{~kb}$ band on a $0.8 \%$ agarose gel. This corresponded with the expected size of the ARO10 gene (http://db.yeastgenome.org) and sequencing of the PCR product confirmed the fidelity of the amplification. The gene product was expressed in $E$. coli as a C-terminal $6 \times$ His variant and was found primarily as soluble protein in the cell-free fraction. ScPPDC-His was purified to homogeneity (Fig. 2) and remained stable for at least 6 months when kept at $-80{ }^{\circ} \mathrm{C}$ in storage buffer. On the basis of SDS/PAGE chromatography, the apparent molecular mass of ScPPDC-His was approximately $66 \mathrm{kDa}$ (Fig. 2). This was at odds with the calculated molecular mass of $72.3 \mathrm{kDa}$ determined from the translated amino acid sequence. However, the ScPPDC sample was clearly larger than an authentic sample of BFDC

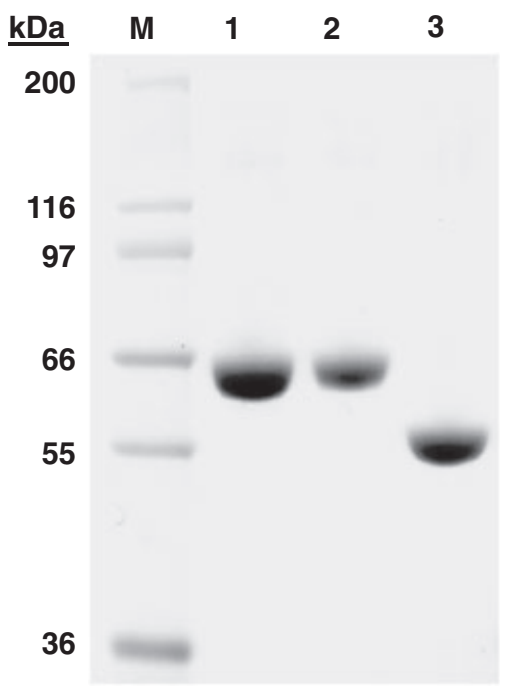

Fig. 2. Purified protein samples were run on a $4-12 \%$ Bis-Tris/Mops-SDS gel. Lanes: 1, Mark $12 \mathrm{MW}$ standards (Invitrogen); 2, ScPPDC-His; 3, ScPPDCE545L-His; 4, BFDC-His. BFDC, a typical ThDP-dependent decarboxylase with a molecular mass of approximately $57 \mathrm{kDa}$, is included for comparison. from $P$. putida $(57.4 \mathrm{kDa})$ when run on the same gel (Fig. 2). Reassuringly, ESI-MS analysis provided a molecular mass of $72122.2 \mathrm{kDa}$. This corresponded well with the calculated molecular mass of ScPPDCHis lacking the two N-terminal residues, Met and Ala (72 $113.6 \mathrm{kDa}$ ). The $\mathrm{N}$-terminal sequence of ScPPDCHis was determined to be PVTIEKFV, corresponding to residues 3-10 of the expected ScPPDC sequence, confirming that the N-terminal Met and Ala were indeed absent. Although cleavage of the terminal Met was not unexpected, the loss of the alanine residue was initially surprising. However, the literature revealed several examples in which the presence of proline as the antepenultimate residue led to the removal of both methionine and alanine. These include interleukin-2 [27] and the bullfrog ribonuclease RNaseRC-4 [28], both expressed in E. coli.

Although ThDP-dependent decarboxylases are generally tetrameric [29], gel filtration of native $S c$ PPDCHis revealed that $S c$ PPDC-His exists in solution primarily as a dimer, with a small proportion $(<15 \%)$ of tetramer (data not shown). Although unusual, this is not without precedent because recent studies have shown that the branched-chain keto acid decarboxylase (KdcA) from Lactococcus lactis is a homodimer both in crystals [30] and solution [31].

The $\mathrm{pH}$ optimum of ScPPDC-His was found to be in the range 6.5-7.0, with activity falling sharply above pH 7.0 (Fig. 3). This is typical for a ThDP-dependent enzyme and likely relates to the $\mathrm{p} K_{\mathrm{a}}$ of the $4^{\prime}$-aminopyridinium group on the cofactor [32]. As a consequence, unless noted otherwise, kinetic characterizations were carried out at $\mathrm{pH}$ 7.0.

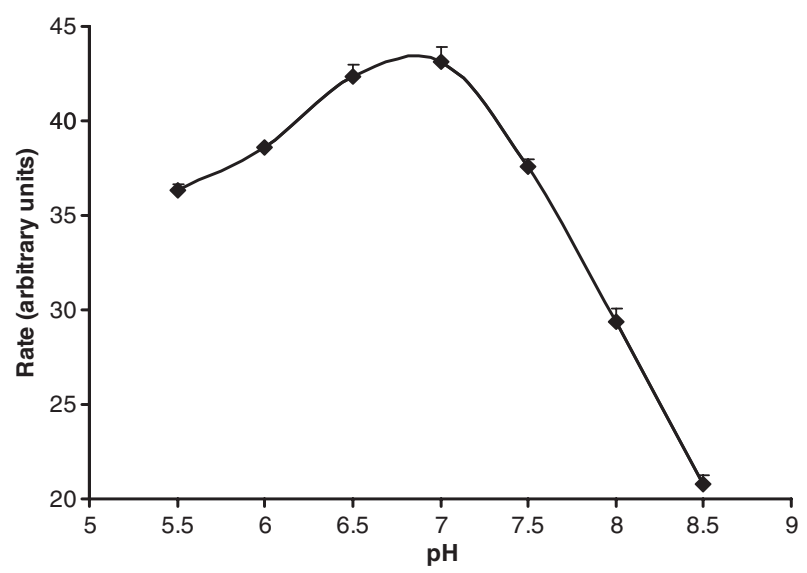

Fig. 3. $\mathrm{pH}$ Screen of ScPPDC-His. The pH optimum of ScPPDC-His was determined as described in the Materials and methods. Each point represents the mean \pm SEM of three separate determinations. 
Although the specific activity of $S c$ PPDC has been measured for several substrates using crude cell extracts [4], to date, there has been no comprehensive examination using the purified enzyme. Accordingly, the kinetic parameters for a selection of 2-keto acids were determined for ScPPDC-His. The 2-keto acids were chosen to provide information on (a) the specificity of the enzyme for the products of amino acid catabolism and (b) the overall substrate specificity of $S c$ PPDC. As shown in Table 1, phenylpyruvic acid (PPA) and indolepyruvic acid (IPyA) were the preferred substrates. On the basis of $K_{\mathrm{m}}$ values, IPyA had higher affinity for the enzyme than PPA, although decarboxylation of the former proceeded more slowly. 4-Hydroxyphenylpyruvate, the substrate derived from tyrosine, had a similar affinity to PPA for ScPPDC-His but was decarboxylated at approximately half the rate.

By comparison, 2-keto acids derived from aliphatic amino acids were much poorer substrates for ScPPDC-His (Table 1). It was notable that much of the reduced activity could be related to substrate binding rather than $k_{\text {cat }}$ effects. With the exception of PPA $\left(20 \mathrm{~s}^{-1}\right), k_{\mathrm{cat}}$ values for the aromatic substrates were approximately $10 \mathrm{~s}^{-1}$. The methionine-derived substrate, 4-methylthio-2-ketobutanoic acid, bound to the enzyme with approximately 20 -fold lower affinity than IPyA but was turned over more quickly, with a $k_{\text {cat }}$ value approaching $8 \mathrm{~s}^{-1}$. A similar result was observed for the non-natural substrate, 2-ketohexanoic acid and the branched-chain 2-keto acid derived from leucine, 4-methyl-2-ketopentanoic acid. The $k_{\text {cat }}$ value for the isoleucine derivative, 3-methyl-2-ketopentanoic acid, was similar to that of its structural isomer but its $K_{\mathrm{m}}$ value was approximately three-fold higher, as well as 30 -fold higher than that for PPA.

The shorter straight chain 2-keto acids (2-ketopentanoic acid, 2-ketobutanoic acid and pyruvic acid) exhibited $K_{\mathrm{m}}$ values approximately 20 - to 100 -fold higher than that for PPA, with the values increasing as the chain length decreased. The $k_{\mathrm{cat}}$ values for the $\mathrm{C} 4, \mathrm{C} 5$ and C6 2-keto acids also decreased as the chain length decreased but, in general, they were broadly similar to those of the other substrates tested. The combination of a smaller side chain and the presence of a 3-methyl substituent resulted in the biggest decrease in $k_{\mathrm{cat}} / K_{\mathrm{m}}$ for a 'natural' substrate. Even then the 80 -fold increase in $K_{\mathrm{m}}$ value for the valine derivative, 3-methyl-2-ketobutanoic acid, suggested that the problem was an inability to bind the substrate rather than problems with turnover. It was not until pyruvate that an order of magnitude decrease in $k_{\text {cat }}$ value was observed.

As described above, ScPPDC was able to utilize aliphatic 2-keto acids, albeit with reduced efficiency. To further explore effect of chain length on substrate specificity, we looked at the reaction of ScPPDC with benzoylformic acid (2-keto-2-phenylethanoic acid) and 2-keto-4-phenylbutanoic acid. These substrates have a phenyl group attached to a $2 \mathrm{C}$ and a $4 \mathrm{C}$ keto acid, respectively. Of the two, benzoylformate was the poorer substrate and it was only possible to collect kinetic data under $V / K$ conditions. Interestingly, 2-keto-4phenylbutanoic acid proved to be the first substrate where the effect of an alteration in chain length was primarily manifested in the $k_{\text {cat }}$ value. For example, although its $K_{\mathrm{m}}$ value was similar to that of PPA and 4-hydroxyphenylpyruvate, 2-keto-4-phenylbutanoate

Table 1. ScPPDC substrate profile. Mean \pm SEM of at least three separate determinations for reactions carried out at $\mathrm{pH} 7.0$. ND, not determined; NAD, no activity detected.

\begin{tabular}{|c|c|c|c|c|c|}
\hline Substrate & Amino acid & $K_{\mathrm{m}}(\mathrm{mm})$ & $k_{\text {cat }}\left(\mathrm{s}^{-1}\right)$ & $k_{\text {cat }} / K_{\mathrm{m}}\left(\mathrm{mm}^{-1} \cdot \mathrm{s}^{-1}\right)$ & $\%^{a}$ \\
\hline Phenylpyruvic acid & Phe & $0.10 \pm 0.01$ & $20 \pm 2.1$ & 200 & 100 \\
\hline Indole-3-pyruvic acid & $\operatorname{Trp}$ & $0.03 \pm 0.01$ & $5.4 \pm 0.3$ & 200 & 100 \\
\hline 4-Hydroxyphenylpyruvic acid & Tyr & $0.09 \pm 0.01$ & $11 \pm 0.8$ & 125 & 63 \\
\hline 4-Methylthio-2-ketobutanoic acid & Met & $0.64 \pm 0.03$ & $7.7 \pm 0.1$ & 12 & 6 \\
\hline 4-Methyl-2-ketopentanoic acid & Leu & $0.90 \pm 0.03$ & $10 \pm 0.1$ & 11 & 6 \\
\hline 3-Methyl-2-ketopentanoic acid & Ile & $3.1 \pm 0.3$ & $11 \pm 0.6$ & 3.5 & 2 \\
\hline 3-Methyl-2-ketobutanoic acid & Val & $8.5 \pm 0.4$ & $19 \pm 0.5$ & 2.2 & 1 \\
\hline 2-Keto-4-phenylbutanoic acid & & $0.09 \pm 0.01$ & $1.7 \pm 0.01$ & 19 & 9 \\
\hline 2-Ketohexanoic acid & & $0.69 \pm 0.01$ & $8.8 \pm 0.1$ & 13 & 6 \\
\hline 2-Ketopentanoic acid & & $2.1 \pm 0.1$ & $5.2 \pm 0.1$ & 2.5 & 1 \\
\hline 2-ketobutanoic acid & & $7.6 \pm 0.6$ & $3.9 \pm 0.1$ & 0.52 & 0.2 \\
\hline Pyruvic acid & & $9.7 \pm 0.1$ & $0.34 \pm 0.01$ & 0.035 & 0.02 \\
\hline Benzoylformic acid & & ND & ND & $0.35^{\mathrm{b}}$ & 0.2 \\
\hline 3-Indoleglyoxylic acid & & NAD & - & - & - \\
\hline
\end{tabular}

${ }^{a}$ Percentage of $k_{\text {cat }} / K_{\mathrm{m}}$ for PPA. ${ }^{\mathrm{b}} V / K$ conditions used. 
was turned over approximately 14-fold and seven-fold more slowly than PPA and 4-hydroxyphenylpyruvate, respectively. Finally, ScPPDC was unable to utilize 3indoleglyoxylic acid as a substrate. Thus, the activity of the enzyme with the indole-derived substrates, 3-indoleglyoxylic acid and IPyA, somewhat paralleled that with benzoylformic acid and PPA.

The ARO10 gene was initially found to be inducible by tryptophan [13] and later by phenylalanine [3], suggesting that $S c$ PPDC is likely to have indole-3-pyruvate and phenylpyruvate decarboxylase activity, respectively. It was also noted that the $A R O 10$ gene was subject to the same regulatory mechanism as the ARO9 gene, which encodes aromatic aminotransferase II [13]. The latter is inducible by all the aromatic amino acids and so it is not entirely unexpected that ScPPDC was found to decarboxylate 4-hydroxyphenylpyruvate (Table 1).

It has been proposed that a single decarboxylase activity was responsible for the catabolism of leucine, methionine and phenylalanine [4]. Conversely, Dickinson et al. [2] reported that ScPPDC contributed only $6 \%$ of the isoleucine flux. The results obtained in the present study show that the efficiency of leucine decarboxylation is only $5 \%$ of that of phenylalanine, suggesting that Dickinson et al. [2] are more likely to be correct. ScPPDC has a relatively low, but measurable, activity with the isoleucine-derived, 3-methyl-2-ketopentanoic acid. This is also consistent with the report that an ARO10 (YDR380w) knockout was able to grow using isoleucine as sole nitrogen source but that, in the absence of other pyruvate decarboxylases, the presence of the ScPPDC was sufficient to allow cell growth. Perpète et al. [9] reported that ScPPDC was essential for the decarboxylation step in methionine catabolism. If it is assumed that, similar to the other amino acids catabolized to fusel alcohols in yeast, methionine was solely catabolized by the Ehrlich pathway, this observation would appear to be at odds with the relatively poor usage of 4-methylthio-2-ketobutanoic acid (Table 1). However, Perpète et al. [9] also demonstrated that Met may be catabolized by transamination to 4-methylthio-2-ketobutanoic acid, then demethiolation, yielding methanethiol and 2-ketobutyrate. Unlike the fusel alcohols, 2-ketobutyrate provides a useful carbon skeleton. Thus, although $S$. cerevisiae may indeed be able to catabolize Met through the Ehrlich pathway via $S c$ PPDC, this route would be less beneficial to the organism which, in turn, is reflected in the relatively low $k_{\text {cat }} / K_{\mathrm{m}}$ value for 4-methylthio-2-ketobutanoic acid.

Overall, these data show that substrates derived from the aromatic amino acids are clearly preferred over those from aliphatic amino acids and confirm that $S c P P D C$, the ARO10 gene product, is an efficient aromatic 2-keto acid decarboxylase, consistent with its proposed in vivo role in phenylalanine and tryptophan catabolism [2]. It may also be involved in tyrosine metabolism but, when alternative pathways are available, it is unlikely to play any significant role in the catabolism of the branched-chain amino acids or of methionine.

\section{Identification and mutation of residues affecting substrate specificity}

There are two histidine residues in the active site of ScPDC located adjacent to each other on a single monomer [33]. This effectively forms a 'HH' motif that is conserved in other PDCs [34,35] and PDC-like decarboxylases such as $\mathrm{KdcA}$, the branched-chain keto acid decarboxylase from L. lactis [30], the indole-3pyruvate decarboxylase from Enterobacter cloacae (EcIPDC) [36] and the PPDC from Azospirillum brasilense (AbPPDC) [37]. The last two enzymes are discussed in more detail below.

CLUSTALW alignment of the sequence of ScPPDC with those of ScPDC, ZmPDC, KdcA and EcIPDC suggested that Ile335, Gln448 and Met624 were likely to shape its substrate binding pocket. This was confirmed using a homology model (Fig. 4A) of ScPPDC obtained from the PHYRE server (http:/www.sbg.bio.ic.ac.uk/phyre/) using the structure of KdcA [Protein Data Bank (PDB): 2VBG] as the template. It is clear from Fig. 4B, which was obtained by superimposing the cofactors of several ' $\mathrm{HH}$ ' motif decarboxylases, that the relative size of the substrates is inversely proportional to the size of the residues. Accordingly, three mutants (I335Y, Q464W and M624W) were prepared with a view to enhancing the activity of ScPPDC towards the shorter chain-length substrates such as pyruvate, and the unbranched substrates, which the wild-type enzyme does not favor (Table 1).

The results, provided in Table 2, confirm that all three residues are important for catalysis by $S c$ PPDC. The $k_{\text {cat }}$ value for reaction of each variant with PPA is lower than that of the wild-type enzyme and, with the exception of Q448W, $K_{\mathrm{m}}$ values are increased and $k_{\text {cat }} / K_{\mathrm{m}}$ values are reduced by almost two orders of magnitude. When viewed as a whole, it is apparent that these mutations have had the most beneficial effect on the decarboxylation of 2-ketopentanoic acid. Each variant has a $K_{\mathrm{m}}$ value less than $1 \mathrm{~mm}$, which is lower than that of the wild-type enzyme, as well as $k_{\text {cat }} / K_{\mathrm{m}}$ values that are greater than that of the wildtype enzyme. This trend does not extend to the shorter 
Fig. 4. (A) Superposition of the homology model of the ScPPDC dimer (orange) and the template (KdcA, PDB: 2VGB, cyan). The left hand side of the figure shows several of the additional loops necessary to account for the $20 \%$ greater molecular mass of the former. (B) Superposition of the putative substrate binding residues of SCPPDC (orange), KdcA (cyan) IPDC (PDB: 1OVM, magenta) and ZmPDC (PDB: 2WVA, yellow). The pyruvate and triazole-ThDP (elemental colors) were from PDB: 2WVA. The dots represent the van der Waals radius of the methyl group of pyruvate.

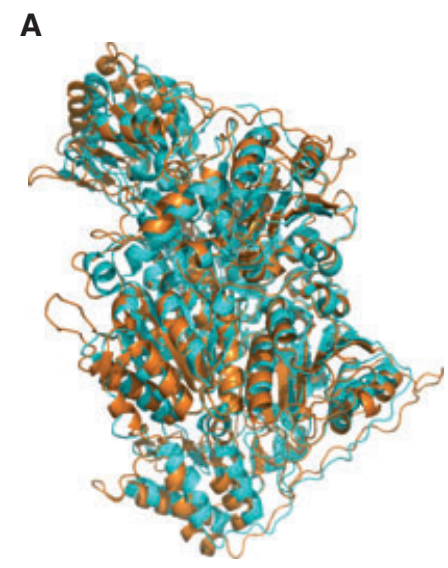

Table 2. Activity of SCPPDC Variants towards short chain and unbranched substrates (mean \pm SD of two or three individual determinations). The data were obtained using the standard assay conditions at $\mathrm{pH}$ 6.0. ND, not determined (assayed under V/K conditions). NAD, no activity detected.

\begin{tabular}{|c|c|c|c|c|c|}
\hline & Wild-type & I335Y & O448W & M624W & I335Y/M624W \\
\hline \multicolumn{6}{|l|}{ Phenylpyruvic acid } \\
\hline$K_{\mathrm{m}}(\mathrm{mm})$ & $0.10 \pm 0.01$ & $0.80 \pm 0.03$ & $0.054 \pm 0.002$ & $0.18 \pm 0.04$ & ND \\
\hline$k_{\text {cat }}\left(\mathrm{s}^{-1}\right)$ & $20 \pm 2.1$ & $0.85 \pm 0.02$ & $4.8 \pm 0.04$ & $1.2 \pm 0.07$ & ND \\
\hline$k_{\mathrm{cat}} / K_{\mathrm{m}}\left(\mathrm{mm}^{-1} \cdot \mathrm{s}^{-1}\right)$ & 200 & $1.1(0.6)^{\mathrm{a}}$ & $89(45)$ & $6.7(3.4)$ & $0.030(0.02)$ \\
\hline \multicolumn{6}{|l|}{ 2-Ketohexanoic acid } \\
\hline$K_{\mathrm{m}}(\mathrm{mm})$ & $0.48 \pm 0.2$ & $0.55 \pm 0.10$ & $0.46 \pm 0.02$ & $0.17 \pm 0.02$ & $1.0 \pm 0.2$ \\
\hline$k_{\text {cat }}\left(\mathrm{s}^{-1}\right)$ & $8.8 \pm 0.6$ & $3.3 \pm 0.04$ & $1.3 \pm 0.1$ & $0.96 \pm 0.03$ & $0.41 \pm 0.06$ \\
\hline$k_{\mathrm{cat}} / K_{\mathrm{m}}\left(\mathrm{mm}^{-1} \cdot \mathrm{s}^{-1}\right)$ & 18 & $6.0(33)$ & $2.8(16)$ & $5.8(32)$ & $0.42(2.3)$ \\
\hline \multicolumn{6}{|l|}{ 2-Ketopentanoic acid } \\
\hline$K_{\mathrm{m}}(\mathrm{mm})$ & $1.7 \pm 0.2$ & $0.63 \pm 0.04$ & $0.54 \pm 0.08$ & $0.37 \pm 0.03$ & $0.96 \pm 0.06$ \\
\hline$k_{\text {cat }}\left(\mathrm{s}^{-1}\right)$ & $2.8 \pm 0.2$ & $2.1 \pm 0.1$ & $2.0 \pm 0.01$ & $0.76 \pm 0.06$ & $0.39 \pm 005$ \\
\hline$k_{\text {cat }} / K_{\mathrm{m}}\left(\mathrm{mm}^{-1} \cdot \mathrm{s}^{-1}\right)$ & 1.6 & $3.4(213)$ & $3.7(231)$ & $2.1(131)$ & $0.40(25)$ \\
\hline \multicolumn{6}{|l|}{ 2-Ketobutanoic acid } \\
\hline$K_{\mathrm{m}}(\mathrm{mm})$ & $7.6 \pm 0.6$ & $6.4 \pm 1.8$ & $7.0 \pm 1.1$ & $5.5 \pm 0.8$ & NAD \\
\hline$k_{\text {cat }}\left(\mathrm{s}^{-1}\right)$ & $3.9 \pm 0.1$ & $0.06 \pm 0.01$ & $2.2 \pm 0.1$ & $0.04 \pm 0.01$ & NAD \\
\hline$k_{\mathrm{cat}} / K_{\mathrm{m}}\left(\mathrm{mm}^{-1} \cdot \mathrm{s}^{-1}\right)$ & 0.51 & $0.009(1.7)$ & $0.315(62)$ & $0.007(1.4)$ & NAD \\
\hline \multicolumn{6}{|l|}{ Pyruvic acid } \\
\hline$K_{\mathrm{m}}(\mathrm{mm})$ & $5.7 \pm 0.1$ & $25 \pm 3$ & $5.5 \pm 0.2$ & $6.4 \pm 0.9$ & ND \\
\hline$k_{\text {cat }}\left(\mathrm{s}^{-1}\right)$ & $0.26 \pm 0.00$ & $0.064 \pm 0.004$ & $0.32 \pm 0.01$ & $0.45 \pm 0.03$ & ND \\
\hline$k_{\mathrm{cat}} / K_{\mathrm{m}}\left(\mathrm{mM}^{-1} \cdot \mathrm{s}^{-1}\right)$ & 0.046 & $0.003(7)$ & $0.06(130)$ & $0.07(152)$ & $0.027(59)$ \\
\hline
\end{tabular}

${ }^{\text {a }}$ Comparison of $k_{\text {cat }} / K_{m}$ with wild-type ScPPDC-His for the corresponding substrate (expressed as a percentage).

chain 2-keto acids, 2-ketobutanoate and pyruvate, where the kinetic constants are broadly similar for each variant. That said, even the latter observation is in sharp contrast to the results for PPA where the individual variants showed significant decreases in $k_{\text {cat }} / K_{\mathrm{m}}$ values. In a previous study, the corresponding variants of the branched-chain decarboxylase from L. lactis, $\mathrm{KdcA}$, were prepared [38]. In those cases, an improvement was observed for the binding of pyruvate but, overall, $k_{\text {cat }} / K_{\mathrm{m}}$ for pyruvate remained well below those obtained for the natural substrate, 3-methyl-2ketopentanoic acid.
Attempts were made to prepare the combinations of double mutants, as well as the triple mutant. Of these, only the I335Y/M624W variant was produced as soluble protein. Although the kinetic data for this variant, also provided in Table 2, indicated that PPA was no longer a viable substrate, there was also little improvement in the utilization of aliphatic substrates. The difficulties with the stability of the double and triple mutants mirrored that observed with KdcA [38]. Although a single site mutant of PDC has been shown to possess excellent 2-ketohexanoate decarboxylase activity [15], the results reported in the present study 
reinforce those of the $\mathrm{KdcA}$ study, which suggest that mutation of single residues is unlikely to produce an 'instant' PDC. It appears that it will be easier to expand the active site to accept larger substrates and, as shown for benzoylformate decarboxylase [16], saturation mutagenesis of at least two residues will be required to shift the substrate preference towards pyruvate. This finding is consistent with the recent study carried out by Steinmetz et al. [39] which showed that, in the ThDP-dependent acetohydroxy acid synthase, two residues act in concert to mediate substrate binding and specificity. Concomitantly, both residues orient substrates and intermediates to ensure optimal alignment of orbitals throughout the reaction [39].

\section{Comparison with other aromatic 2-keto acid decarboxylases}

To date, there has been only one detailed characterization of a phenylpyruvate decarboxylase, the $p d c$ gene product from the nitrogen-fixing bacterium, A. brasilense. Initially, this enzyme was identified as an indole3-pyruvate decarboxylase because it played a central role in the formation of indole acetic acid, the most abundant naturally occurring auxin [40,41]. However, subsequent analysis showed that its substrate spectrum was markedly different to that of the homologous IPDC from E. cloacae [19]. For example, in addition to IPyA, the EcIPDC was able to decarboxylate both benzoylformate and pyruvate [42,43], but not PPA $[40,42]$. Furthermore, there was no evidence for substrate activation of EcIPDC [42]. Conversely, the $A$. brasilense enzyme showed a ten-fold greater $k_{\mathrm{cat}} / K_{\mathrm{m}}$ for PPA than for IPyA, no activity with benzoylformate, and substrate activation was observed with IPyA and several other substrates [19]. Ultimately, this led to the classification of the $A$. brasilense ipdC gene product as a phenylpyruvate decarboxylase ( $A b P P D C$ ).

The data provided in Table 1 indicate that $S c P P D C$ is quite different to both $A b P P D C$ and $E c I P D C$ in that it is able to decarboxylate PPA and IPyA, essentially with equal efficiency. It is more like $A b P P D C$ in that it can also decarboxylate 4-phenyl-2-ketobutanoic acid and 2-ketohexanoic acid, although it does so without evidence for substrate activation. On the other hand, ScPPDC can decarboxylate 3- and 4-methyl-2 ketopentanoic acid, as well as benzoylformate, whereas $A b$ PPDC cannot [19]. In short, the activities of the two phenylpyruvate decarboxylases are similar yet different, and both are distinct from those of EcIPDC.

A multiple sequence alignment of a suite of known and putative bacterial PPDCs and IPDCs showed that the sequences divided into two clades, indicating that they have derived from at least two different ancestors [19]. The sequences in one clade all possessed a glutamic acid residue at the position corresponding to residue 468 of EcIPDC. In the second clade, which included $A b P P D C$, in most cases, the glutamate was replaced by a leucine. Consequently, it was speculated that the specificity for IPyA or PPA might be determined by the presence of a glutamic acid or a leucine, respectively, in this position. Expansion of this alignment to include yeast PPDC homologs shows that the yeast sequences cluster with the 'IPDC' group, all having Glu at this position (data not shown). To explore this issue further, the Glu545Leu variant of ScPPDC was prepared, and its kinetic parameters with the two substrates, PPA and IPyA, were determined. A comparison of these and the parameters of the wild-type enzyme are presented in Table 3.

$S c$ PPDC-E545L was unstable in the buffer used for the IPyA assay and thus was assayed in the standard buffer but with the loss of NADH monitored at $366 \mathrm{~nm}$. Unexpectedly, this variant showed evidence of allosteric activation by both PPA and IPyA, with Hill coefficients of 1.8 and 2.1, respectively. By contrast, the wild-type enzyme showed little evidence of allosteric activation with Hill coefficients approaching 1 for both substrates. Comparison of $S_{0.5}$ values showed that both substrates bound with higher affinity to the E545L variant. For PPA, the affinity increased almost 30-fold, although this was accompanied by a decrease in $k_{\text {cat }}$ value of more than 700 -fold. For IPyA, a threefold increase in binding affinity was observed, with a concomitant 38 -fold decrease in $k_{\text {cat }}$ value. Overall, although the ScPPDC-E545L variant is a much poorer decarboxylase than the wild-type enzyme, it could be argued that, with a $15: 9$ ratio of $k_{\text {cat }} / S_{0.5}$, the substrate preference has been switched to favor IPyA. Of

Table 3. Activity of E545L ScPPDC-His. All data were obtained at pH 7.0 and were analyzed using the simplified Hill equation.

\begin{tabular}{lll}
\hline & Wild-type & E545L $^{\mathrm{a}}$ \\
\hline Phenylpyruvic acid & & \\
$S_{0.5}(\mu \mathrm{M})$ & $97 \pm 8$ & $3.4 \pm 0.3(29)$ \\
$k_{\text {cat }}\left(\mathrm{s}^{-1}\right)$ & $22 \pm 2.1$ & $0.03 \pm 0.002(730)$ \\
$k_{\text {cat }} / S_{0.5}\left(\mathrm{~mm}^{-1} \cdot \mathrm{s}^{-1}\right)$ & 226 & $8.8(25)$ \\
$n_{\mathrm{h}}$ & $1.06 \pm 0.06$ & $1.84 \pm 0.14$ \\
Indole-3-pyruvic acid & & \\
$S_{0.5}(\mu \mathrm{M})$ & $27 \pm 3$ & $9.6 \pm 1.7(2.8)$ \\
$k_{\text {cat }}\left(\mathrm{s}^{-1}\right)$ & $5.3 \pm 0.2$ & $0.14 \pm 0.01(38)$ \\
$k_{\text {cat }} / S_{0.5}\left(\mathrm{~mm}^{-1} \cdot \mathrm{s}^{-1}\right)$ & 196 & $15(13)$ \\
$n_{\mathrm{h}}$ & $1.15 \pm 0.13$ & $2.13 \pm 0.27$ \\
\hline
\end{tabular}

${ }^{a}$ The fold decrease from wild-type enzyme is shown in parentheses. 
course, this is the opposite result to that expected if a leucine residue in this position was truly indicative of an enzyme being a PPDC [19].

Although it is possible to argue about whether there is a true switch in substrate preference, the data provided in Table 3 show conclusively that the E545L variant has (a) enhanced affinity for both PPA and IPyA and (b) evidence for allosteric activation that was not present in the wild-type enzyme. What is not clear are the reasons for those observations. ScPPDC Glu545 has well characterized equivalents in EcIPDC (Glu468), ZmPDC (Glu473) and ScPDC (Glu477) and forms part of a Glu-Asp-His triad that has long been associated with the various protonation-deprotonation steps in the decarboxylation reaction [43-47]. Early modeling studies suggested that Glu477 (ScPDC) participates in the decarboxylation of the 2-lactyl-thiamin diphosphate intermediate, as well as in the protonation of the carbanion-enamine intermediate [44]. These predictions were recently confirmed experimentally using a combination of $\mathrm{CD}$ and NMR spectroscopy using the E473D and E473Q variants of ZmPDC [47]. It is interesting to note that these variants both showed an approximately 1000 -fold decrease in the value of $k_{\text {cat }}$, similar to that observed for the E477Q variant of ScPDC [46], whereas the E468D variant of EcIPDC showed an approximately 25 -fold decrease in the value of $k_{\text {cat }}$ [43]. In all cases, the $K_{\mathrm{m}}$ values were essentially unaffected by the mutation $[43,46,47]$. In our case, the E545L variant showed, depending on the substrate, decreases in $k_{\text {cat }}$ reminiscent of both pyruvate and IPyA decarboxylases, although these decreases were accompanied by a concomitant decrease in $K_{\mathrm{m}}$ values for PPA and IPyA (Table 3). Given that replacement of this glutamic acid residue results in relatively large (two or three orders of magnitude) changes in $k_{\text {cat }}$ values, it is noteworthy that $A b P P D C$, which has a leucine at the corresponding position, has a $k_{\text {cat }}$ value of $5.6 \mathrm{~s}^{-1}$ [19]. This value is broadly similar to the $k_{\text {cat }}$ values of EcIPDC $\left(3.9 \mathrm{~s}^{-1}\right)$ [42], ScPDC $\left(36 \mathrm{~s}^{-1}\right)$ [46] and $S c$ PPDC $\left(20 \mathrm{~s}^{-1}\right)$ (Table 1) with their natural substrates. Clearly, AbPPDC has been able to adapt, and it was suggested that this was achieved by Leu462 providing an increase in the hydrophobicity of the active site, thereby stabilizing zwitterionic intermediates [37]. Nevertheless, the catalytic mechanism of a ThDPdependent decarboxylase requires a number of proton transfer steps, and these are often mediated through a network of water molecules. Removal of a hydrophilic residue such as E545 has the potential to disrupt any hydrogen bonding/water molecule network, which will also result in a reduction in $k_{\text {cat }}$ values. It is conceivable that, for the ScPPDC E545L variant, the increase in hydrophobicity is insufficient to compensate for the loss of an acid-base catalyst, or the disruption of a hydrogen bond network, although it does result in an increase in binding affinity for PPA and IPyA.

One question still remains: why does the E545L variant show allosteric activation? Again, the answer may lie with $A b P P D C$. It has been proposed that, rather than the typical Glu-Asp-His motif, AbPPDC has an Asp-Asp-His catalytic triad in which Asp282 replaces the glutamic acid residue. Moreover, it is only after the substrate binds at the regulatory site that Asp282 moves into the active site to form the catalytic triad [48]. In Glu334, ScPPDC has a residue occupying a similar position to Asp282 and which, potentially, may act as a surrogate for Glu545 in the catalytic triad (albeit with a reduced effect). The observation of allosteric activation would then arise from a requirement for the substrate to bind to effect the correct positioning of Glu334 for catalysis. Of course, this explanation also requires a second, regulatory, substrate binding site. In $A b P P D C$, the second phenylpyruvate is held tightly by charged interactions of its carboxylate with two arginine residues (Arg60 andArg215) and a hydrogen bond to the backbone of Ala397, while the phenyl ring forms a cation $-\pi$ interaction with $\operatorname{Arg} 214$ [48]. Intriguingly, superposition of the ScPPDC model and the AbPPDC-3dThDP-PPA (PDB: 2Q5O) indicates that a similar site may be present in the former. In Arg88 and Arg267, ScPPDC has positional counterparts for Arg60 and Arg215, respectively. The amide of Ser464 is similarly located to that of Ala397in AbPPDC and, although ScPPDC has no direct counterpart to Arg214, there is a lysine residue, Lys461, that could rotate in and perform a similar function to Arg214. Clearly, this explanation is speculative, although it does provide a basis for our ongoing investigations. As an aside, previous results reported by Meyer et al. [47] also suggest that the enamine of the E545L variant is likely to be long lived, raising the possibility that this variant may carry out more efficient carboligation reactions than the wild-type enzyme. This too is being explored in our continuing studies.

In summary, we have demonstrated that the $S$. cerevisiae ARO10 gene product comprises an efficient phenylpyruvate decarboxylase likely playing a prominent role in the catabolism of aromatic, but not aliphatic, amino acids. Furthermore, we have reinforced previous studies concluding that it will take more than point mutations to significantly alter substrate specificity in ThDP-dependent decarboxylases. Finally, we have that shown that it is unlikely that replacement of a glutamic acid by leucine leads to discrimination between the two substrates, phenylpyruvate and 
indolepyruvate, although it did lead to unexpected allosteric activation.

\section{Materials and methods}

\section{Reagents}

S. cerevisiae genomic DNA was obtained from Novagen (EMD4Biosciences, Gibbstown, NJ, USA). PCR primers were purchased from Integrated DNA Technologies (Coralville, IA, USA). The sequences of the primers are provided in Table S1. Nickel-nitrilotriacetic acid resin was obtained from Qiagen (Valencia, CA, USA). Substrates were purchased from Sigma-Aldrich (St Louis, MO, USA), Acros (Morris Plains, NJ, USA) and ChemBridge (San Diego, CA, USA). Buffer and assay components were from Sigma-Aldrich or Fisher Scientific (Pittsburgh, PA, USA) and were of the highest commercially-available quality.

\section{Amplification}

The coding region for SCPPDC (ARO10) was amplified $S$. cerevisiae genomic DNA using the primer pair PPDCNdeI and PPDC-XhoI, which inserted $N d e I$ and XhoI sites at the $5^{\prime}$ and $3^{\prime}$ ends, respectively, of the ScPPDC gene. The amplified fragment was gel-purified then ligated into pCRBlunt (Invitrogen, Carlsbad, CA, USA) and the resulting plasmid transformed into chemically-competent E. coli TOP10 cells. Plasmid DNA from the transformants was purified and the NdeI-XhoI fragment inserted into a modified $\mathrm{pET} 17 \mathrm{~b}$ vector (Novagen) to give the expression vector, pET17PPDC-His, thereby permitting Ni-chelate affinity purification of ScPPDC-His. The fidelity of the amplification and vector construction was confirmed by sequencing of pET17PPDC-His (University of Michigan DNA Sequencing Core Facility, Ann Arbor, MI, USA).

\section{Mutagenesis}

Single residue variants of ScPPDC-His were prepared by the QuikChange method (Stratagene, Agilent Technologies Inc., Santa Clara, CA, USA) using pET17PPDC-His and the primers listed in Table S1. The presence of the changed nucleotides was screened by restriction digestion and confirmed by sequencing.

\section{Purification}

ScPPDC-His and its variants were overexpressed in E. coli BL21(DE3)pLysS cells (Novagen). ScPPDC-His expression was induced at room temperature with isopropyl thio- $\beta$-Dgalactoside and the cultures grown for $20 \mathrm{~h}$. All subsequent ScPPDC-His purification procedures were performed at $4{ }^{\circ} \mathrm{C}$. Cells were pelleted by centrifugation and resuspended in buffer $\mathrm{A}(50 \mathrm{mM} \mathrm{NaPO}, \mathrm{pH} 8.0,300 \mathrm{~mm} \mathrm{NaCl})$ containing $5 \mathrm{~mm}$ imidazole, then frozen at $-80{ }^{\circ} \mathrm{C}$ overnight. The frozen cells were thawed and incubated for $30 \mathrm{~min}$ with DNase $\left(5 \mu \mathrm{g} \cdot \mathrm{mL}^{-1}\right)$ and lysozyme $\left(0.2 \mathrm{mg} \cdot \mathrm{mL}^{-1}\right)$ then disrupted by sonication $(3 \times 30 \mathrm{~s}$ bursts, with $1 \mathrm{~min}$ rest between bursts). Clarified cell-free extract was obtained by two centrifugation steps of $30 \mathrm{~min}$ at $20000 \mathrm{~g}$.

The cell-free extract was applied to a nickel-nitrilotriacetic acid (Qiagen) column attached to a Biologic LC system (Bio-Rad, Hercules, CA, USA) and equilibrated with buffer A. The column was extensively washed with buffer A and weakly-bound proteins eluted with buffer B (buffer A containing $20 \mathrm{~mm}$ imidazole). ScPPDC-His was eluted with buffer C (buffer A containing $250 \mathrm{~mm}$ imidazole). Fractions containing ScPPDC-His were pooled, then desalted and exchanged into storage buffer (50 mM $\mathrm{KPO}_{4}, \mathrm{pH} 7.0,1 \mathrm{~mm}$ $\mathrm{MgSO}_{4}, 0.5 \mathrm{~mm}$ ThDP, $10 \%$ glycerol) using an Econo-Pac DG10 desalting column (Bio-Rad). The purified protein was concentrated (Amicon Ultra, Millipore, Billerica, MA, USA) and stored at $-80^{\circ} \mathrm{C}$.

The purity of the ScPPDC-His was verified by SDS/PAGE. The protein concentration was determined spectrophotometrically using $\varepsilon_{280}=71260 \mathrm{M}^{-1} \cdot \mathrm{cm}^{-1}$ calculated with PROT PARAM (http://expasy.org/tools/protparam. html) or by the Bradford assay using BSA as standard.

\section{Assay}

The decarboxylation activity of $S c$ PPDC-His on a range of aromatic and aliphatic 2-keto acids was monitored at $30^{\circ} \mathrm{C}$ by a coupled assay described previously [38]. The reaction mixture contained (in $1 \mathrm{~mL}$ ): $100 \mathrm{mM} \mathrm{KPO}_{4}$ buffer (pH 6.0 or 7.0); $1 \mathrm{~mm} \mathrm{MgSO}_{4} ; 0.5 \mathrm{~mm}$ ThDP; $200 \mu \mathrm{M}$ NADH; 0.05-0.25 U horse liver alcohol dehydrogenase or yeast alcohol dehydrogenase and varying concentrations of 2keto acid. The reaction was initiated by addition of ScPPDC-His $\left(2.5-190 \mu \mathrm{g} \cdot \mathrm{mL}^{-1}\right)$ and the loss of NADH was monitored at $340 \mathrm{~nm}$. Stock solutions of the 2-keto acids were usually prepared in assay buffer $\left(100 \mathrm{mM} \mathrm{KPO}_{4}\right.$, $1 \mathrm{mM} \mathrm{MgSO}_{4}, 0.5 \mathrm{mM} \mathrm{ThDP}$ ) and the $\mathrm{pH}$ adjusted to 6.0 or 7.0 as required. When necessary, ScPPDC-His was diluted into assay buffer containing $1 \mathrm{mg} \cdot \mathrm{mL}^{-1}$ BSA.

Monitoring the decarboxylation of IPyA had been reported by Schütz et al. [42] to be problematic. Consequently, IPyA decarboxylation by ScPPDC was determined in an assay buffer containing $10 \mathrm{~mm}$ Mes buffer $(\mathrm{pH} 6.5)$, $1 \mathrm{~mm} \mathrm{MgSO}_{4}$ and $0.5 \mathrm{~mm}$ ThDP, with the reaction being monitored at $366 \mathrm{~nm}$ to reduce interference as a result of the high absorbance of IPyA at $340 \mathrm{~nm} \mathrm{[19].} \mathrm{IPyA} \mathrm{stock}$ solutions were prepared in assay buffer and incubated for 45-60 $\mathrm{min}$ at room temperature to ensure maximal conversion from the enol to the keto tautomer [42]. The E545L variant precipitated in this buffer and therefore was assayed under the standard conditions used for all other substrates, although the reaction was monitored at $366 \mathrm{~nm}$. 
Analysis of enzyme kinetics data was performed with the ENZYME KINETICS package within SIGMAPLOT (Systat Software, Inc., Chicago, IL, USA), using the single-substrate model and Michaelis-Menten and Hill analysis.

The $\mathrm{pH}$ optimum of ScPPDC-His for the decarboxylation of PPA was determined by the coupled assay described above, using a ScPPDC-His concentration of $2.5 \mu \mathrm{g} \cdot \mathrm{mL}^{-1}$ and PPA at $0.62 \mathrm{~mm}$. The $\mathrm{pH}$ determined in this screen was used for all subsequent experiments unless otherwise stated.

\section{Size-exclusion chromatography}

The molecular mass of native ScPPDC-His was determined by size exclusion chromatography on a Sephacryl S-200 HR Column $(1.6 \times 94 \mathrm{~cm})$, equilibrated with $50 \mathrm{~mm}$ $\mathrm{NaPO}_{4}, 150 \mathrm{~mm} \mathrm{NaCl}(\mathrm{pH}$ 7.0). The void volume of the column was determined with Blue Dextran, and the elution volume of ScPPDC-His compared with those of ferritin (440 kDa), aldolase (158 kDa), conalbumin (75 kDa) and albumin (43 kDa) (GE Healthcare HMW Gel Filtration Standards; GE Healthcare, Milwaukee, WI, USA). In addition, P putida BFDC was included as a standard because its native molecular mass and multimeric form is well-established [17].

\section{MS and N-terminal sequencing}

ScPPDC-His was exchanged into $50 \mathrm{~mm}$ Mops, $1 \mathrm{~mm}$ $\mathrm{MgSO}_{4}, 0.5 \mathrm{~mm}$ ThDP (pH 7.0) for analysis by LC-MS. $\mathrm{N}$-terminal sequencing was performed following electrophoretic transfer of $\mathrm{ScPPDC}-\mathrm{His}$ to a poly(vinylidene difluoride) membrane. Both were performed at the University of Michigan Protein Structure Facility (Ann Arbor, MI, USA).

\section{Acknowledgements}

This work was supported by the National Science Foundation (Grant EF-0425719 to M.J.M.) and by the University of Michigan Undergraduate Research Opportunity Program (UROP to R.P.T.).

\section{References}

1 Hazelwood LA, Daran JM, van Maris AJ, Pronk JT \& Dickinson JR (2008) The Ehrlich pathway for fusel alcohol production: a century of research on Saccharomyces cerevisiae metabolism. Appl Environ Microbiol 74, 2259-2266.

2 Dickinson JR, Salgado LE \& Hewlins MJ (2003) The catabolism of amino acids to long chain and complex alcohols in Saccharomyces cerevisiae. J Biol Chem 278, $8028-8034$.
3 Vuralhan Z, Morais MA, Tai SL, Piper MD \& Pronk JT (2003) Identification and characterization of phenylpyruvate decarboxylase genes in Saccharomyces cerevisiae. Appl Environ Microbiol 69, 4534-4541.

4 Vuralhan Z, Luttik MA, Tai SL, Boer VM, Morais MA, Schipper D, Almering MJ, Kotter P, Dickinson JR, Daran JM et al. (2005) Physiological characterization of the ARO10-dependent, broad-substrate-specificity 2-oxo acid decarboxylase activity of Saccharomyces cerevisiae. Appl Environ Microbiol 71, 3276-3284.

5 Atsumi S, Hanai T \& Liao JC (2008) Non-fermentative pathways for synthesis of branched-chain higher alcohols as biofuels. Nature 451, 86-89.

6 Zhang K, Sawaya MR, Eisenberg DS \& Liao JC (2008) Expanding metabolism for biosynthesis of nonnatural alcohols. Proc Natl Acad Sci USA 105, 20653-20658.

7 Dickinson JR, Lanterman MM, Danner DJ, Pearson BM, Sanz P, Harrison SJ \& Hewlins MJ (1997) A ${ }^{13} \mathrm{C}$ nuclear magnetic resonance investigation of the metabolism of leucine to isoamyl alcohol in Saccharomyces cerevisiae. J Biol Chem 272, 26871-26878.

8 ter Schure EG, Flikweert MT, van Dijken JP, Pronk JT \& Verrips CT (1998) Pyruvate decarboxylase catalyzes decarboxylation of branched-chain 2-oxo acids but is not essential for fusel alcohol production by Saccharomyces cerevisiae. Appl Environ Microbiol 64, 1303-1307.

9 Perpete P, Duthoit O, De Maeyer S, Imray L, Lawton AI, Stavropoulos KE, Gitonga VW, Hewlins MJ \& Dickinson JR (2006) Methionine catabolism in Saccharomyces cerevisiae. FEMS Yeast Res 6, 48-56.

10 Kellermann E, Seeboth PG \& Hollenberg CP (1986) Analysis of the primary structure and promoter function of a pyruvate decarboxylase gene (PDC1) from Saccharomyces cerevisiae. Nucleic Acids Res 14, 8963-8977.

11 Seeboth PG, Bohnsack K \& Hollenberg CP (1990) pdc1(0) mutants of Saccharomyces cerevisiae give evidence for an additional structural PDC gene: cloning of PDC5, a gene homologous to PDC1. J Bacteriol 172, 678-685.

12 Hohmann S (1991) Characterization of PDC6, a third structural gene for pyruvate decarboxylase in Saccharomyces cerevisiae. J Bacteriol 173, 7963-7969.

13 Iraqui I, Vissers S, Andre B \& Urrestarazu A (1999) Transcriptional induction by aromatic amino acids in Saccharomyces cerevisiae. Mol Cell Biol 19, 3360-3371.

14 Godard P, Urrestarazu A, Vissers S, Kontos K, Bontempi G, van Helden J \& Andre B (2007) Effect of 21 different nitrogen sources on global gene expression in the yeast Saccharomyces cerevisiae. Mol Cell Biol 27, 3065-3086.

15 Siegert P, McLeish MJ, Baumann M, Iding H, Kneen MM, Kenyon GL \& Pohl M (2005) Exchanging the substrate specificities of pyruvate decarboxylase from Zymomonas mobilis and benzoylformate decarboxylase 
from Pseudomonas putida. Protein Eng Des Sel 18, 345 357.

16 Yep A \& McLeish MJ (2009) Evolution of a benzoylformate decarboxylase variant with enhanced pyruvate decarboxylase activity. Biochemistry 48, 8387-8395.

17 Hasson MS, Muscate A, McLeish MJ, Polovnikova LS, Gerlt JA, Kenyon GL, Petsko GA \& Ringe D (1998) The crystal structure of benzoylformate decarboxylase at $1.6 \AA$ resolution: diversity of catalytic residues in thiamin diphosphate-dependent enzymes. Biochemistry 37, 9918-9930.

18 Ward OP \& Singh A (2005) Enzyme biotransformation involving $\alpha$-ketoacid decarboxylases. In Microorganisms for Industrial Enzymes and Biocontrol (Mellado E \& Barredo J-L eds), pp. 83-94. Research Signpost, Trivandrum, India.

19 Spaepen S, Versees W, Gocke D, Pohl M, Steyaert J \& Vanderleyden J (2007) Characterization of phenylpyruvate decarboxylase, involved in auxin production of Azospirillum brasilense. J Bacteriol 189, 7626-7633.

20 Asakawa T, Wada H \& Yamano T (1968) Enzymatic conversion of phenylpyruvate to phenylacetate. Biochim Biophys Acta 170, 375-391.

21 Barrowman MM \& Fewson CA (1985) Phenylglyoxylate decarboxylase and phenylpyruvate decarboxylase from Acinetobacter calcoaceticus. Curr Microbiol 12, 235-240.

22 Guo ZZ (1999) Asymmetric acyloin condensation catalyzed by phenylpyruvate decarboxylase. Tetrahedron Asymmetry 10, 4667.

23 de Boer L, Harder W \& Dijkhuizen L (1988) Phenylalanine and tyrosine metabolism in the facultative methyltroph Nocardia sp. 239. Arch Microbiol 149, 459-465.

24 Schneider S, Mohamed ME \& Fuchs G (1997) Anaerobic metabolism of L-phenylalanine via benzoyl-CoA in the denitrifying bacterium Thauera aromatica. Arch Microbiol 168, 310-320.

25 Kishore G, Sugumaran M \& Vaidyanathan CS (1976) Metabolism of DL-(+/-)-phenylalanine by Aspergillus niger. J Bacteriol 128, 182-191.

26 Altschul SF, Madden TL, Schaffer AA, Zhang J, Zhang Z, Miller W \& Lipman DJ (1997) Gapped BLAST and PSI-BLAST: a new generation of protein database search programs. Nucleic Acids Res 25, 3389-3402.

27 Ben-Bassat A, Bauer K, Chang SY, Myambo K, Boosman A \& Chang S (1987) Processing of the initiation methionine from proteins: properties of the Escherichia coli methionine aminopeptidase and its gene structure. $J$ Bacteriol 169, 751-757.

28 Liao YD, Jeng JC, Wang CF, Wang SC \& Chang ST (2004) Removal of N-terminal methionine from recombinant proteins by engineered $E$. coli methionine aminopeptidase. Protein Sci 13, 1802-1810.
29 Duggleby RG (2006) Domain relationships in thiamine diphosphate-dependent enzymes. Acc Chem Res 39, 550-557.

30 Berthold CL, Gocke D, Wood MD, Leeper FJ, Pohl M \& Schneider G (2007) Structure of the branched-chain keto acid decarboxylase ( $\mathrm{KdcA}$ ) from Lactococcus lactis provides insights into the structural basis for the chemoselective and enantioselective carboligation reaction. Acta Crystallogr D Biol Crystallogr 63, 1217-1224.

31 Gocke D, Nguyen CL, Pohl M, Stillger T, Walter L \& Muller M (2007) Branched-chain keto acid decarboxylase from Lactococcus lactis (KdcA), a valuable thiamine diphosphate-dependent enzyme for assymmetric C-C bond formation. Adv Synth Catal 349, 1425-1435.

32 Nemeria N, Korotchkina L, McLeish MJ, Kenyon GL, Patel MS \& Jordan F (2007) Elucidation of the chemistry of enzyme-bound thiamin diphosphate prior to substrate binding: defining internal equilibria among tautomeric and ionization states. Biochemistry 46, 10739-10744.

33 Arjunan P, Umland T, Dyda F, Swaminathan S, Furey W, Sax M, Farrenkopf B, Gao Y, Zhang D \& Jordan F (1996) Crystal structure of the thiamin diphosphatedependent enzyme pyruvate decarboxylase from the yeast Saccharomyces cerevisiae at $2.3 \AA$ resolution. J Mol Biol 256, 590-600.

34 Dobritzsch D, Konig S, Schneider G \& Lu G (1998) High resolution crystal structure of pyruvate decarboxylase from Zymomonas mobilis. Implications for substrate activation in pyruvate decarboxylases. $J$ Biol Chem 273, 20196-20204.

35 Kutter S, Wille G, Relle S, Weiss MS, Hubner G \& Konig S (2006) The crystal structure of pyruvate decarboxylase from Kluyveromyces lactis. Implications for the substrate activation mechanism of this enzyme. FEBS J 273, 4199-4209.

36 Schutz A, Sandalova T, Ricagno S, Hubner G, Konig S \& Schneider G (2003) Crystal structure of thiamindiphosphate-dependent indolepyruvate decarboxylase from Enterobacter cloacae, an enzyme involved in the biosynthesis of the plant hormone indole-3-acetic acid. Eur J Biochem 270, 2312-2321.

37 Versées W, Spaepen S, Vanderleyden J \& Steyaert J (2007) The crystal structure of phenylpyruvate decarboxylase from Azospirillum brasilense at $1.5 \mathrm{~A}$ resolution. Implications for its catalytic and regulatory mechanism. FEBS J 274, 2363-2375.

38 Yep A, Kenyon GL \& McLeish MJ (2006) Determinants of substrate specificity in KdcA, a thiamin diphosphate-dependent decarboxylase. Bioorg Chem 34, 325-336.

39 Steinmetz A, Vyazmensky M, Meyer D, Barak ZE, Golbik R, Chipman DM \& Tittmann K (2010) Valine 375 and phenylalanine 109 confer affinity and specificity for pyruvate as donor substrate in acetohydroxy acid 
synthase isozyme II from Escherichia coli. Biochemistry 49, 5188-5199.

40 Koga J, Adachi T \& Hidaka H (1992) Purification and characterization of indolepyruvate decarboxylase. A novel enzyme for indole-3-acetic acid biosynthesis in Enterobacter cloacae. J Biol Chem 267, 15823-15828.

41 Costacurta A, Keijers V \& Vanderleyden J (1994) Molecular cloning and sequence analysis of an Azospirillum brasilense indole-3-pyruvate decarboxylase gene. Mol Gen Genet 243, 463-472.

42 Schutz A, Golbik R, Tittmann K, Svergun DI, Koch MH, Hubner G \& Konig S (2003) Studies on structurefunction relationships of indolepyruvate decarboxylase from Enterobacter cloacae, a key enzyme of the indole acetic acid pathway. Eur J Biochem 270, 2322-2331.

43 Schutz A, Golbik R, Konig S, Hubner G \& Tittmann $\mathrm{K}$ (2005) Intermediates and transition states in thiamin diphosphate-dependent decarboxylases. A kinetic and NMR study on wild-type indolepyruvate decarboxylase and variants using indolepyruvate, benzoylformate, and pyruvate as substrates. Biochemistry 44, 6164-6179.

44 Lobell M \& Crout DHG (1996) Pyruvate decarboxylase: a molecular modeling study of pyruvate decarboxylation and acyloin formation. $\mathrm{J} \mathrm{Am}$ Chem Soc 118, 1867-1873.

45 Chang AK, Nixon PF \& Duggleby RG (1999) Aspartate-27 and glutamate-473 are involved in catalysis by Zymomonas mobilis pyruvate decarboxylase. Biochem $J$ 339, 255-260.

46 Liu M, Sergienko EA, Guo F, Wang J, Tittmann K, Hubner G, Furey W \& Jordan F (2001) Catalytic acid- base groups in yeast pyruvate decarboxylase. 1. Sitedirected mutagenesis and steady-state kinetic studies on the enzyme with the D28A, H114F, H115F, and E477Q substitutions. Biochemistry 40, 7355-7368.

47 Meyer D, Neumann P, Parthier C, Friedemann R, Nemeria N, Jordan F \& Tittmann K (2010) Double duty for a conserved glutamate in pyruvate decarboxylase: evidence of the participation in stereoelectronically controlled decarboxylation and in protonation of the nascent carbanion/enamine intermediate. Biochemistry 49, 8197-8212.

48 Versées W, Spaepen S, Wood MD, Leeper FJ, Vanderleyden J \& Steyaert J (2007) Molecular mechanism of allosteric substrate activation in a thiamine diphosphate-dependent decarboxylase. J Biol Chem 282, 35269-35278.

\section{Supporting information}

The following supplementary material is available:

Table S1. ScPPDC-His PCR primers.

This supplementary material can be found in the online version of this article.

Please note: As a service to our authors and readers, this journal provides supporting information supplied by the authors. Such materials are peer-reviewed and may be re-organized for online delivery, but are not copy-edited or typeset. Technical support issues arising from supporting information (other than missing files) should be addressed to the authors. 